\title{
MONITORING OF WINE PROCESS AND PREDICTION OF ITS PARAMETERS WITH MID-INFRARED SPECTROSCOPY
}

\author{
CANAN CANAL and BANU OZEN ${ }^{1}$ \\ Izmir Institute of Technology, Department of Food Engineering, Urla-Izmir, Turkey
}

${ }^{1}$ Corresponding author. TEL:

90-232-750-6319; FAX: 90-232-750-6196;

EMAIL: banuozen@iyte.edu.tr

Received for Publication March 30, 2015

Accepted for Publication August 6, 2015

doi:10.1111/jfpe. 12280

\begin{abstract}
It was aimed to predict the chemical (ethanol, glycerol, organic acids, titratable acidity, ${ }^{\circ}$ Brix, sugars, total phenolic and anthocyanin content) and microbiological parameters of red, rose and white wines during their processing from must to bottling using mid-infrared (IR) spectroscopy in combination with one of the multivariate statistical analysis techniques, partial least square (PLS) regression. Various spectral filtering techniques were employed before PLS regression analysis of mid-IR data. The best results were obtained from the second-order derivation for the chemical parameters except for alcohols. PLS models developed for the prediction of some of the chemical parameters have $R^{2}$ values greater than 0.9 , with low root mean square error values; however, prediction of microbial population from mid-IR spectroscopy did not provide accurate results. IR spectroscopic and chemical-chromatographic data were also used to investigate the differences between processing steps, and principal component analysis allowed clear separation of the beginning of the process from the rest.
\end{abstract}

\section{PRACTICAL APPLICATIONS}

Monitoring of the wine process from must to final product is necessary for better control of the process and the quality. As a rapid and a minimum waste-producing technique, mid-IR spectroscopy in combination with chemometric methods could allow prediction of several chemical parameters simultaneously. Therefore, any problems that could be encountered during wine processing could be determined and interfered in a short time.

\section{INTRODUCTION}

Wine is a complex mixture of various chemical compounds. Water and ethanol are the major compounds of wine, whereas sugars, organic acids, phenolic compounds, salts, glycerol, aliphatic and aromatic alcohols are the minor components that are taking part in the flavor and color formation. Concentration of these minor components is of great importance in terms of both quality assurance for winemaking industries and consumer preferences (Tarantilis et al. 2008).

Several factors such as variety of grape used in wine production, ripeness of grape, geographic origin, vintage and viticultural and vinification techniques have considerable influence on compositional variations of wine (Serban et al. 2004). Wine production requires strict monitoring of a series of parameters for a long period of time. The diversity of parameters and the complexity of this product require fast, selective, precise and sensitive methods of analysis. In order to produce high-quality wine, the changes in composition should be periodically controlled at every stage of grape and wine as the chemical and microbiological composition of grape and wine alters from the start of grape ripening to the harvest, the maturity, the grape acceptance, in the course of wine production and aging. Fast and accurate analytical techniques are desirable for monitoring and screening of the product and process. Fourier transform infrared (FTIR) spectroscopy is a powerful technique for identifying types of chemical bonds in a molecule. Intensity of peaks in infrared (IR) spectra is also proportional to the concentrations of the constituents of the samples analyzed. Multivariate statistical analysis techniques improved the applicability of mid-IR spectroscopy to several food products to differentiate them according to their chemical 
properties or to determine their different quality parameters quantitatively. Several studies were also performed in the literature about monitoring of wine composition using various IR spectroscopic techniques during the fermentation process (Esti et al. 2003; Cozzolino et al. 2004; Di Egidio et al. 2010). However, whole wine process was not investigated for the applicability of mid-IR spectroscopy for the determination of various chemical parameters simultaneously with minimum sample preparation and with minimum laboratory waste generation. FTIR spectroscopy combined with chemometrics could enable a simultaneous determination of a wide range of enological parameters and provide rapid, repeatable and nondestructive analysis of wine with minimum sample preparation and chemical consumption. In addition, information about the complete composition of wine can be supplied from the FTIR spectra (Edelman et al. 2001), which can provide monitoring of all chemical changes in wine during its process. Furthermore, there is no study in literature regarding the detection of microbial population of wine with infrared spectroscopic techniques.

The purpose of this study was to predict the parameters (ethanol glycerol, organic acids, titratable acidity, ${ }^{\circ} \mathrm{Brix}$, sugars, total phenol and anthocyanin content, yeast population) that are critical in wine processing by mid-IR spectroscopy in combination with multivariate statistical analysis of data obtained during various stages of red, rose and white wine processing. In addition, differentiation of process stages with regard to chemical parameters and spectroscopic data was investigated.

\section{MATERIALS AND METHODS}

\section{Samples}

A total of seven wine samples (two white wines: Muscat and Chardonnay; one rose wine: Sangiovese; four red wines: Syrah, Merlot, Cabernet Sauvignon and Boğazkere) were provided by a commercial local winery (Urla Winery, UrlaIzmir, Turkey). Grapes used for wine processes were sourced from vineyards also in Urla region of Izmir. All wine fermentation processes were carried out using stainless steel vessels with the capacity of 45-90 L. The fermentation processes of red wines were conducted at 18-19C, whereas those of rose and white wines were carried out at 15-16C using active dry yeast inoculum (Saccharomyces cerevisiae) in 20 and $25 \mathrm{~g} / \mathrm{L}$ concentrations, respectively. Sampling was performed at some critical points during the production of wines to glass bottles (Fig. 1), and a total of 35 wine samples were obtained at the end of the process as shown in the diagram. Samples were transferred to the laboratory at the sampling day. Because of a problem in the processing of Boğazkere, sampling of this wine was stopped after the third stage of production; therefore, data were only collected before this stage of this particular wine sample. All chemical and microbiological analyses of the samples that were stored in the Schott bottles at $4 \mathrm{C}$ were performed within 1-2 months. The samples that were not analyzed at the same day were stored at $-20 \mathrm{C}$ until usage. All parameters were evaluated at least twice.

\section{Total Phenolic Content}

Total phenolic content (TPC) of wine samples was determined according to Folin-Ciocalteu micromethod modified from total phenol analysis (Slinkard and Singleton 1977), and the results were expressed in terms of gallic acid equivalent (mg GAE/L).

\section{Anthocyanin Determination}

The total anthocyanin content of red wines was determined using $\mathrm{pH}$ differential method (Jungmin et al. 2005), which is based on UV-vis spectrophotometric measurement of reversible color change of anthocyanin pigment at different wavelengths (520 and $700 \mathrm{~nm}$ ).

\section{Titratable Acidity, pH and ${ }^{\circ}$ Brix Measurements}

The titratable acidity measurements of wine samples were performed using the modified AOAC method 962.12 (AOAC 2000). The $\mathrm{pH}$ of wine samples was measured using a calibrated pH meter (WTW Series, Inolab, Weilheim, Germany) according to AOAC method (960.19, AOAC 2000). The refractive index (RI) (total soluble solids) measurements of wine samples were carried out with a calibrated refractometer (Re50, Mettler Toledo, Columbus, $\mathrm{OH}$ ).

\section{Organic Acid, Sugar, Glycerol and Ethanol Content}

High performance liquid chromatography (HPLC) analysis based on the method described by Castellari et al. (2000) was used for the analysis of the main organic compounds sugars, glycerol and ethanol - in musts and wines. An HPX$87 \mathrm{H}(300 \times 7.8 \mathrm{~mm}, 9 \mu \mathrm{m})$ column (Bio-Rad Laboratories, Hercules, CA) attached to the guard column $(30 \times 4.6 \mathrm{~mm})$ was employed for the separation of the compounds. Twenty microliters of wine sample was injected to the HPLC system (Perkin Elmer 200, Waltham, MA), and the column oven was set to $45 \mathrm{C}$. A total of $0.045 \mathrm{~N} \mathrm{H}_{2} \mathrm{SO}_{4}$ with $6 \%$ acetonitrile was used as a mobile phase at a flow rate of $0.5 \mathrm{~mL} / \mathrm{min}$.

For the determination of organic acids, sugars, glycerol and ethanol, the samples were cleaned through $\mathrm{C} 18$ cartridges to eliminate the phenolic components in red and 


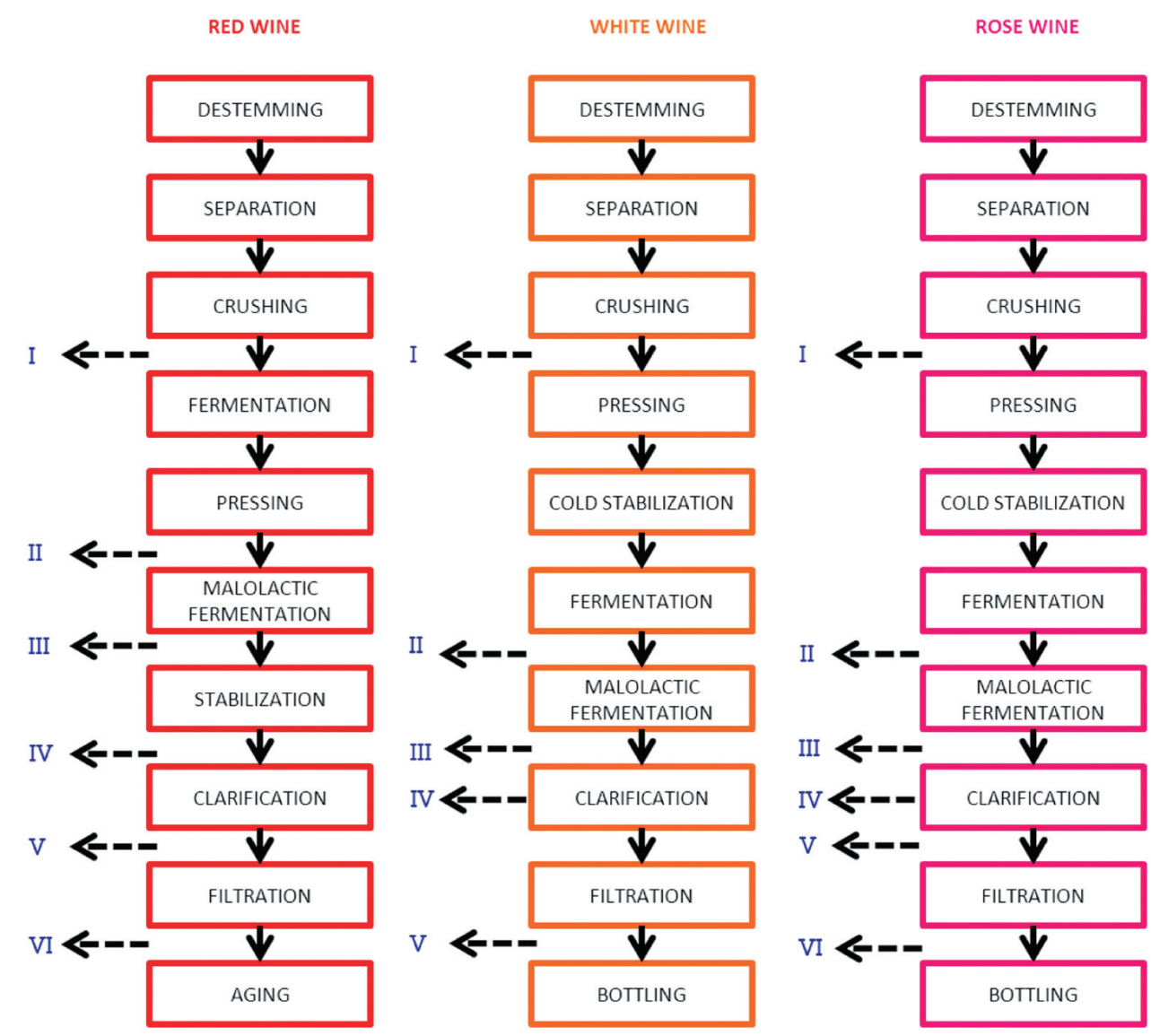

FIG. 1. THE PROCESSES OF RED, WHITE AND ROSE WINES AND THE SAMPLING SITES DURING WINE PROCESS

rose wines as explained in the OIV method (OIV 2009). The cartridges were conditioned with $10 \mathrm{~mL}$ of methanol, and then with $10 \mathrm{~mL}$ of water. Then, $8 \mathrm{~mL}$ of red and rose wine samples was passed through the column. The first $3 \mathrm{~mL}$ was discarded, and the rest was collected. This was followed by 10 -fold dilution with the mobile phase and filtering prior to injection. White wines were only diluted with the mobile phase by 10 -fold and then filtered. Sugars and organic acids commonly found in must and wine were used as the standards in this study. Standards (acetic acid, citric acid, $\alpha$-ketoglutaric acid, fumaric acid, lactic acid, malic acid, pyruvic acid, succinic acid, tartaric acid, glucose and fructose) were purchased from a commercial source (SigmaAldrich, Munich, Germany). The detection of sugar, ethanol and glycerol was performed by RI detector, whereas organic acids were detected by diode array detector $(210 \mathrm{~nm})$. The identification of organic acids, sugars and alcohols was confirmed using integrated peak areas according to external standard calibration.

\section{Mid-Infrared Spectroscopy}

Measurements were performed with an FTIR spectrometer (Perkin Elmer Spectrum 100) within the range of 4,000-
$650 \mathrm{~cm}^{-1}$ wave number. This equipment has a horizontal attenuated total reflectance accessory with $\mathrm{ZnSe}$ crystal $\left(45^{\circ}\right.$ trough plate) and deuterated triglycine sulfate detector. The scanning was carried out at $4 \mathrm{~cm}^{-1}$ resolution with 64 scans for each spectrum and $0.50 \mathrm{~cm} / \mathrm{s}$ scan speed. After each wine scanning, crystal was cleaned with ethanol, and distilled water and dried under nitrogen flow.

\section{Microbial Enumeration}

The microbiological analysis of the must and wine samples was carried out in this study according to OIV method (OIV 2009). S. cerevisiae (NRRL Y-139, ATCC 2366) was used as a positive control as it has been used in the winery for inoculation of all wines for fermentation process. The enumeration of the wine samples was performed with serial 10 -fold dilutions in $0.1 \%$ peptone water, with three replicates. Inoculation was carried out into yeast extract peptone dextrose agar medium (10 g/L yeast extract, $20 \mathrm{~g} / \mathrm{L}$ peptone, $20 \mathrm{~g} / \mathrm{L}$ dextrose and $20 \mathrm{~g} / \mathrm{L}$ agar) followed by incubation at $26 \mathrm{C}$ for $48 \mathrm{~h}$. All of the suspected yeast colonies were counted (colony-forming unit/mL) and evaluated directly under phase contrast microscope. 


\section{Multivariate Statistical Analysis}

The data from the FTIR spectrometer were analyzed using multivariate statistical analysis techniques with SIMCA software (SIMCA P-10.5, Umetrics Inc., Umea, Sweden). Principal component analysis (PCA) was performed using FTIR spectral data and the results of chemical analysis in order to see whether the clustering of wine samples obtained from different steps in the process is possible or not. The data matrix composed of wine samples (observations) and all data (variables) are used to classify the wine samples into disjoint data classes based on the similarities among the members of the same data class (Bauer et al. 2008).

Partial least square (PLS) regression analysis was used to construct the calibration models that enable prediction of chemical and chromatographic parameters of wine samples from FTIR spectral data. This method allows obtaining information about the parameter that is under the interest for the spectral interval by constructing a prediction model (Fernández and Agosin 2007). Spectral filtering techniques such as wavelet compression of spectra (WCS), wavelet in combination with orthogonal signal correction (WOSC) and orthogonal signal correction in combination with wavelet (OSCW), first- and second-order derivation were all employed before the PLS regression analysis. Because of the limited number of samples, the data were not divided into calibration and validation sets. Instead, the cross-validation technique was used to assess the performance of the model. Cross-validation evaluates the data by excluding selected samples in the PLS regression model and then building a model for the remaining samples. All samples in the PLS model are excluded once by this procedure then the error values between predicted and calculated response values were used to calculate the root mean square of error (RMSE) and $R^{2}$ cross-validation $\left(R_{\mathrm{cv}}^{2}\right)$.

\section{RESULTS AND DISCUSSIONS}

\section{Measured Chemical Characteristics of Wines during Processing and Their Statistical Analysis}

The wine samples studied in this research were generally selected from wines of well-known grape varieties grown in Turkey. The results of the chemical analysis of wine samples during the process are listed in Tables 1 and 2. TPC of white wines varied from 117.33 to $541.78 \mathrm{mg} / \mathrm{L}$, whereas red wine samples have TPC in the range of $388.44-8,951.78 \mathrm{mg} / \mathrm{L}$ and anthocyanin content of $12.02-287.22 \mathrm{mg} / \mathrm{L}$. It was observed that both the total phenol and anthocyanin contents at the beginning of the red wine process were much lower than those in the middle of the process. Among the red wine samples, Syrah and Merlot have the highest total phenol $(8,118.4$ and $7,707.3 \mathrm{mg} / \mathrm{L})$ and anthocyanin value ( 163.7 and 129.6 eq.mg/L) in the final product. TPC decreased for all wines in the bottling step, while in terms of anthocyanin content the decrease was observed only in Cabernet. Reported TPC values are higher compared with values given in the literature. However, these values are for the wines before bottling while values in the literature are for bottled and stored wines. As it is well known, phenolic compounds are easily oxidized and could be lost during bottling and storage.

The titratable acidity and $\mathrm{pH}$ values of all wines showed a lower diversity than the other properties (data not shown). Referring to the ${ }^{\circ}$ Brix value, it is observed that sugar concentration is at its highest value at the beginning of the process for all wines while it decreased in further processing steps.

The analysis of organic acids allows controlling the evolution of acidity during different steps of winemaking process (alcoholic fermentation, malolactic fermentation, aging process, etc.). The level of tartaric acid is a parameter of critical control in the stabilization of wine. The results of organic acid analysis show that the concentration of each organic acid was changing during the process for all wine samples (Table 1). Among them, succinic, lactic and tartaric acids were present in the samples in much higher amounts than the others. The initial value of glucose and fructose ranged from 53 to 116 and 68 to $131 \mathrm{~g} / \mathrm{L}$, respectively (Table 2). Ethanol concentration at the end of the process varied from 17 to $21 \% \mathrm{v} / \mathrm{v}$, and glycerol content was about 6-11 g/L. Di Egidio et al. (2010) studied near-IR and mid-IR spectroscopy to monitor red wine fermentation, and found that the initial values of glucose and fructose ranged between $97-130$ and $106-141 \mathrm{~g} / \mathrm{L}$, respectively, whereas ethanol concentration at the end of the process varied between 99 and $127 \mathrm{~g} / \mathrm{L}$ and glycerol content was about $10 \mathrm{~g} / \mathrm{L}$. In a study performed with German wines, alcohol and glycerol ranges were found as 7.4-14\% (v/v) and 5.20$27.80 \mathrm{~g} / \mathrm{L}$, respectively (Patz et al. 2004). The wines used in our study seem to have similar chemical properties with previous studies; however, the ethanol content at the end of the process is higher.

In this study, all wine samples were scanned through the FTIR spectrometer and several chemical parameters of wine were determined with traditional chemical analysis techniques. The difference of the initial process step from further steps can easily be observed from FTIR spectra of a selected wine (Merlot) during the process (Fig. 2). Assignment of absorption bands in FTIR spectra of wines could be found in the literature (Fernández and Agosin 2007; Lachenmeier 2007). As it can be seen from Fig. 2, there is a significant difference between the first and the later stages of wine process. Differences between the absorption intensity of the bands around $3,600-3,200 \mathrm{~cm}^{-1}$ and 1,700 
TABLE 1. ORGANIC ACID CONTENTS OF WINE SAMPLES THROUGH THE PROCESS

\begin{tabular}{|c|c|c|c|c|c|c|c|c|c|c|}
\hline Wine variety & Process steps & CA & TA & $A-C A$ & MA & PA & SA & LA & FA & $A A$ \\
\hline \multirow[t]{5}{*}{ Muscat } & 1 & 245.77 & $1,161.07$ & 160.8 & $1,977.74$ & 6.17 & 59.75 & 441.46 & 40.27 & 1.03 \\
\hline & $\|$ & 145.61 & 834.12 & 33.52 & $1,739.22$ & 19.76 & $1,040.97$ & 392.39 & 15.75 & 157.56 \\
\hline & III & 186.52 & 101.35 & 10.60 & 887.09 & 11.98 & 306.38 & 348.27 & 25.46 & 211.12 \\
\hline & IV & 295.07 & 402.82 & 33.97 & $1,299.27$ & 15.63 & 585.88 & 437.52 & 16.21 & 233.12 \\
\hline & V & 476.06 & 362.97 & 23.62 & $1,457.01$ & 13.61 & 419.63 & 396.93 & 23.78 & 267.68 \\
\hline \multirow[t]{4}{*}{ Chardonnay } & I & 47.92 & $1,579.68$ & 103.34 & $1,447.22$ & 7.46 & 386.48 & 260.27 & 6.74 & 5.54 \\
\hline & $\|$ & 485.09 & 590.76 & 76.84 & $2,510.52$ & 20.56 & 672.42 & 534.18 & 37.23 & 476.53 \\
\hline & III & 140.88 & 404.39 & 179.02 & $1,835.35$ & 33.45 & $1,335.01$ & 403.4 & 25.56 & 298.64 \\
\hline & IV & 309.91 & 607.34 & 82.98 & $2,123.84$ & 12.9 & 805.06 & 411.89 & 35.21 & 222.69 \\
\hline \multirow[t]{6}{*}{ Sangiovese } & I & 166.04 & $1,427.09$ & 57.47 & $2,259.52$ & 3.96 & 329.71 & 196.27 & 9.03 & 39.33 \\
\hline & $\|$ & 456.6 & 916.20 & 177.85 & $3,740.14$ & 18.98 & $1,690.4$ & 411.2 & 48.97 & 196.39 \\
\hline & III & 462.48 & 775.84 & 183.84 & $3,479.84$ & 7.88 & $1,537.20$ & 400.59 & 40.85 & 186.21 \\
\hline & IV & 401.63 & 672.57 & 165.93 & $2,968.43$ & 8.75 & $1,226.13$ & 386.91 & 37.98 & 198.47 \\
\hline & V & 483.3 & 266.99 & 176.82 & $3,610.06$ & 11.44 & $1,555.64$ & 443.34 & 41.5 & 234.86 \\
\hline & $\mathrm{Vl}$ & 478.62 & 295.21 & 206.63 & $3,510.75$ & 11.75 & $1,590.04$ & 443.91 & 42.09 & 260.39 \\
\hline \multirow[t]{6}{*}{ Syrah } & I & 249.89 & 713.85 & 1.78 & $2,706.48$ & 5.53 & $5,706.94$ & 187.91 & 9.55 & 101.97 \\
\hline & $\|$ & 511.58 & 142.83 & 187.05 & $2,719.15$ & 81.39 & $6,463.66$ & 384.85 & 72.12 & 426.30 \\
\hline & III & 688.11 & 23.51 & 201.04 & $2,487.81$ & 71.98 & $7,414.10$ & 464.30 & 105.56 & 465.84 \\
\hline & IV & 603.92 & 51.08 & 226.71 & $2,244.46$ & 29.16 & $7,465.83$ & 891.07 & 93.58 & 670.43 \\
\hline & V & 327.02 & 86.51 & 206.22 & 460.34 & 1.68 & $7,317.12$ & $1,472.58$ & 33.26 & 893.96 \\
\hline & $\mathrm{Vl}$ & 217.95 & 24.11 & 88.325 & 132.021 & 0.99 & $2,949.07$ & 828.58 & 10.73 & 267.1 \\
\hline \multirow[t]{6}{*}{ Merlot } & I & 8.88 & 474.34 & 5.92 & 633.25 & 2.50 & 196.23 & 255.62 & 6.62 & 47.16 \\
\hline & $\|$ & 410.98 & $1,011.24$ & 100.63 & $1,234.50$ & 52.63 & $1,703.39$ & 820.62 & 41.05 & 748.64 \\
\hline & III & 496.28 & $1,414.57$ & 124.29 & $1,074.28$ & 23.12 & $1,798.72$ & $1,043.45$ & 44.62 & 751.69 \\
\hline & IV & 225.78 & $1,250.06$ & 94.69 & 395.44 & 17.88 & $1,692.23$ & $1,817.84$ & 13.3 & $1,089.99$ \\
\hline & V & 572.82 & 592.22 & 104.16 & 295.95 & 22.63 & $1,774.16$ & $1,634.81$ & 9.75 & $1,079.69$ \\
\hline & $\mathrm{Vl}$ & 214.89 & $1,467.41$ & 235.36 & 283.11 & 8.57 & $1,365.81$ & $1,844.62$ & 9.13 & 477.6 \\
\hline \multirow{5}{*}{$\begin{array}{l}\text { Cabernet } \\
\text { sauvignon }\end{array}$} & 1 & 566.87 & $1,554.78$ & 1,044 & $1,611.74$ & 100.25 & $3,902.17$ & 569.38 & 57.30 & 446.03 \\
\hline & $\|$ & 66.44 & 370.77 & 185.14 & 500.29 & 10.847 & $4,429.41$ & $1,304.54$ & 12.83 & $1,422.27$ \\
\hline & III & 450.36 & 318.93 & 152.06 & 667.61 & 13.07 & $6,327.96$ & $1,456.63$ & 11.96 & $2,012.2$ \\
\hline & IV & 305.13 & 468.13 & 85.97 & 405.76 & 79.31 & $4,368.5$ & $1,570.03$ & 17.9 & $1,769.65$ \\
\hline & V & 145.85 & 53,962 & 63.44 & 202.53 & 50.69 & $4,482.71$ & $1,334.11$ & 12.46 & $1,116.30$ \\
\hline \multirow[t]{3}{*}{ Boğazkere } & I & 361.8 & $1,212.02$ & 25.66 & 598.06 & 41.38 & $1,885.03$ & 640.14 & 22.21 & 210.22 \\
\hline & $\|$ & 424.2 & 556.29 & 44.60 & 924.78 & 4.98 & $2,247.94$ & 625.32 & 23.56 & 763.97 \\
\hline & III & 757.29 & $1,067.4$ & 35.53 & 166.63 & 3.19 & $2,370.91$ & $1,445.80$ & 10.47 & 918.8 \\
\hline
\end{tabular}

The results show the averages of multiple measurements.

AA, acetic acid concentrations (mg/L); A-CA, alpha chetoglutaric acid; CA, citric acid; FA, fumaric acid; LA, lactic acid; MA, malic acid; PA, pyruvic acid; SA, succinic acid; TA, tartaric acid.

$1,600 \mathrm{~cm}^{-1}$ could be attributed to $-\mathrm{OH}$ stretching that may come from alcohol and water. Although there is an absorption band at $3,000 \mathrm{~cm}^{-1}$ arising from C-H stretching of $-\mathrm{CH}_{3}$ and $-\mathrm{CH}_{2}$ groups, same peak is not visible for the must sample after the first stage of processing. Peaks at $1,820-1,670 \mathrm{~cm}^{-1}$ and $1,150-1,070 \mathrm{~cm}^{-1}$ due to the absorption bands of $\mathrm{C}-\mathrm{O}$ and $\mathrm{C}-\mathrm{C}$ could be associated with ethanol and organic compounds produced during the fermentation process (Di Egidio et al. 2010). In addition, must spectrum in the fingerprint region $\left(1,565-965 \mathrm{~cm}^{-1}\right)$ is different than the later stages.

PCA was performed using FTIR spectral data and chemical-chromatographic parameters separately in order to see whether the classification of wine samples obtained from different steps in the process is possible or not accord- ing to the chemical characteristics in different processing stages. Developed PCA models using FTIR and chemical data have 15 principal components (PCs) and $R^{2}$ of 0.998 , and three PCs and 0.765 as $R^{2}$ value, respectively. The PCA score plot obtained from the FTIR data allows clear separation of the beginning of the process (first step for all wines) from the rest of the process steps regardless of the wine type. Similar results were obtained from the PCA score plot of the chemical-chromatographic parameters (Fig. 3a). According to the chemical results, rose wine and each white wine form their own clusters in the PCA score plot after the first stage of processing. Therefore, varietal separation was observed for rose and white wines for the later stages of processing, but there was no separation in terms of processing steps. However, Cabernet and Merlot and bottled Syrah 
TABLE 2. SUGAR, ALCOHOL, TOTAL PHENOL, ANTHOCYANIN CONTENTS AND YEAST POPULATION OF WINE SAMPLES THROUGH THE PROCESS

\begin{tabular}{|c|c|c|c|c|c|c|c|c|}
\hline Wine variety & Process steps & Glu & Fru & Gly & Eth & TP & Ant & Yeast (cfu/mL) \\
\hline \multirow[t]{5}{*}{ Muscat } & I & $92,452.15$ & $109,858.1$ & 100.28 & 11.36 & 328.44 & - & $3.3 \times 10^{4}$ \\
\hline & $\|$ & $1,647.44$ & $5,991.77$ & $8,576.12$ & 20.33 & 314.0 & - & $1 \times 10^{2}$ \\
\hline & III & $6,209.46$ & $37,967.03$ & $5,310.85$ & 17.74 & 517.33 & - & $6.7 \times 10^{4}$ \\
\hline & IV & $8,911.86$ & $47,929.84$ & $5,854.05$ & 18.44 & 351.78 & - & $<10$ \\
\hline & V & $7,544.79$ & $45,582.11$ & $6,231.13$ & 17.58 & 541.78 & - & $4.7 \times 10^{1}$ \\
\hline \multirow[t]{4}{*}{ Chardonnay } & I & $77,346.44$ & $91,246.66$ & 569.33 & 8.56 & 197.33 & - & $1.1 \times 10^{2}$ \\
\hline & $\|$ & $8,206.59$ & $58,877.65$ & $8,171.02$ & 18.09 & 117.33 & - & $1.4 \times 10^{7}$ \\
\hline & III & $2,080.53$ & $12,236.01$ & $9,912.05$ & 21.14 & 301.78 & - & $5.2 \times 10^{6}$ \\
\hline & IV & $1,783.49$ & 680.64 & $9,027.19$ & 20.48 & 256.22 & - & $1.2 \times 10^{4}$ \\
\hline \multirow[t]{6}{*}{ Sangiovese } & I & $61,361.24$ & $68,869.20$ & $1,723.68$ & 7.77 & 285.11 & 24.41 & $1.4 \times 10^{4}$ \\
\hline & $\|$ & $1,758.85$ & $1,046.61$ & $10,215.19$ & 21.3 & 170.67 & 13.03 & $2.5 \times 10^{5}$ \\
\hline & III & $1,833.92$ & $1,304.66$ & $9,228.56$ & 20.85 & 206.22 & 14.80 & $5.5 \times 10^{5}$ \\
\hline & IV & $1,654.52$ & 812.4 & $10,464.87$ & 18.47 & 272.89 & 8.32 & $1.3 \times 10^{4}$ \\
\hline & V & $1,936,736$ & 797.33 & $9,477.61$ & 18.91 & 307.33 & 7.55 & $5 \times 10^{1}$ \\
\hline & $\mathrm{Vl}$ & $1,673.19$ & 632.73 & $9,540.78$ & 19.39 & 337.33 & 6.45 & $3.4 \times 10^{2}$ \\
\hline \multirow[t]{6}{*}{ Syrah } & I & $103,422.7$ & $120,621.4$ & 409.56 & 8.13 & 388.44 & 12.02 & $3.1 \times 10^{5}$ \\
\hline & $\|$ & $1,627.18$ & 642.09 & $11,477.81$ & 22.32 & $3,618.44$ & 273.19 & $4.1 \times 10^{5}$ \\
\hline & III & $1,689.31$ & 649.71 & $12,579.93$ & 22.03 & $5,985.11$ & 202.39 & $7 \times 10^{4}$ \\
\hline & IV & $1,621.02$ & 624.49 & $6,622.3$ & 14.62 & $7,829.56$ & 133.59 & $6.3 \times 10^{4}$ \\
\hline & V & $1,643.85$ & $1,044.16$ & $12,181.34$ & 21.87 & $7,396.22$ & 149.62 & $9.6 \times 10^{2}$ \\
\hline & $\mathrm{Vl}$ & $1,680.31$ & 832.76 & $11,024.91$ & 21.15 & $8,118.44$ & 163.65 & $1.8 \times 10^{5}$ \\
\hline \multirow[t]{6}{*}{ Merlot } & I & $116,198.2$ & $131,167.1$ & $1,414.50$ & 8.40 & 498.44 & 27.39 & $1.8 \times 10^{5}$ \\
\hline & $\|$ & $2,292.76$ & $16,155.45$ & $10,275.24$ & 19.28 & $3,385.11$ & 90.18 & $1.1 \times 10^{5}$ \\
\hline & III & $1,975.87$ & $5,106.93$ & $6,038.36$ & 14.42 & $4,985.11$ & 76.15 & $8.2 \times 10^{4}$ \\
\hline & IV & $1,718.18$ & $1,750.97$ & $9,763.81$ & 18.87 & $8,107.33$ & 92.18 & $1.6 \times 10^{3}$ \\
\hline & V & $1,706.17$ & $1,283.57$ & $11,962.45$ & 22.19 & $8,951.78$ & 116.89 & $8.3 \times 10^{1}$ \\
\hline & $\mathrm{Vl}$ & $1,714.44$ & 811.22 & $11,007.54$ & 20.68 & $7,707.33$ & 129.58 & $1 \times 10^{3}$ \\
\hline \multirow[t]{5}{*}{ Cabernet } & I & $53,808.59$ & $101,828.86$ & $4,500.66$ & 11.98 & 92.85 & 4.25 & $7.3 \times 10^{6}$ \\
\hline & $\|$ & $1,877.41$ & $16,105.89$ & $9,840.11$ & 19.81 & 134.93 & 5.07 & $5.1 \times 10^{5}$ \\
\hline & III & $1,980.2$ & $18,643.46$ & $10,506.85$ & 20.43 & 123.57 & 3.87 & $1.3 \times 10^{4}$ \\
\hline & IV & $1,690.03$ & $11,331.6$ & $10,824.23$ & 20.69 & 131.59 & 4.25 & $4.3 \times 10^{2}$ \\
\hline & V & $1,654.23$ & $3,199.64$ & $10,499.78$ & 19.73 & 99.53 & 3.61 & $2.3 \times 10^{2}$ \\
\hline \multirow[t]{3}{*}{ Boğazkere } & 1 & $5,821.61$ & $34,335.75$ & $6,228.81$ & 172.33 & 4.71 & - & $1.8 \times 10^{7}$ \\
\hline & $\|$ & $1,642.23$ & 629.52 & $8,290.89$ & 208.40 & 3.45 & - & $2 \times 10^{3}$ \\
\hline & III & $1,733.82$ & 664.22 & $9,274.06$ & 287.22 & 3.15 & - & $2 \times 10^{1}$ \\
\hline
\end{tabular}

The results show the averages of multiple measurements.

Ant, anthocyanin (cyd-3-glu eq.mg/L) concentrations and yeast population (cfu/mL); cfu, colony-forming unit; Eth, ethanol (\% v/v); Fru, fructose (ppm); Glu, glucose (ppm); Gly, glycerol (mg/L); TP, total phenol (mg gallic acid/L).

red wines gathered together separately from the rest of the clusters of rose and white wines. According to PCA loading plot (Fig. 3b) of the chemical-chromatographic data, the beginning of the wine process differs from the others especially in terms of ${ }^{\circ}$ Brix value, glucose and fructose content.

\section{Prediction of Chemical Parameters by IR Spectroscopy}

PLS is one of the most commonly used regression technique in multivariate statistical analysis and it is employed to show the relation between FTIR data and the results of chemical and chromatographic analysis in this study. Therefore, it could be possible to develop equations for predicting some enological parameters of wine samples relating chemical parameters to spectroscopic data.

The chemical results including TPC, anthocyanin content, titratable acidity, $\mathrm{pH},{ }^{\circ} \mathrm{Brix}$ value and chromatographic results including organic acids, sugar, ethanol and glycerol content were analyzed in combination with FTIR spectral data. Because the acidity terms are highly related to each other, the titratable acidity, $\mathrm{pH}$ and organic acid results were analyzed jointly. Similarly, ${ }^{\circ}$ Brix, sugar and alcohol results were modeled in separate groups. However, TPC and anthocyanin content were analyzed individually. In anthocyanin regression analysis, only the results of red and rose wines were used as anthocyanin pigment only exists in red grapes. 
FIG. 2. FOURIER TRANSFORM INFRARED SPECTRA OF MERLOT WINES DURING PROCESS

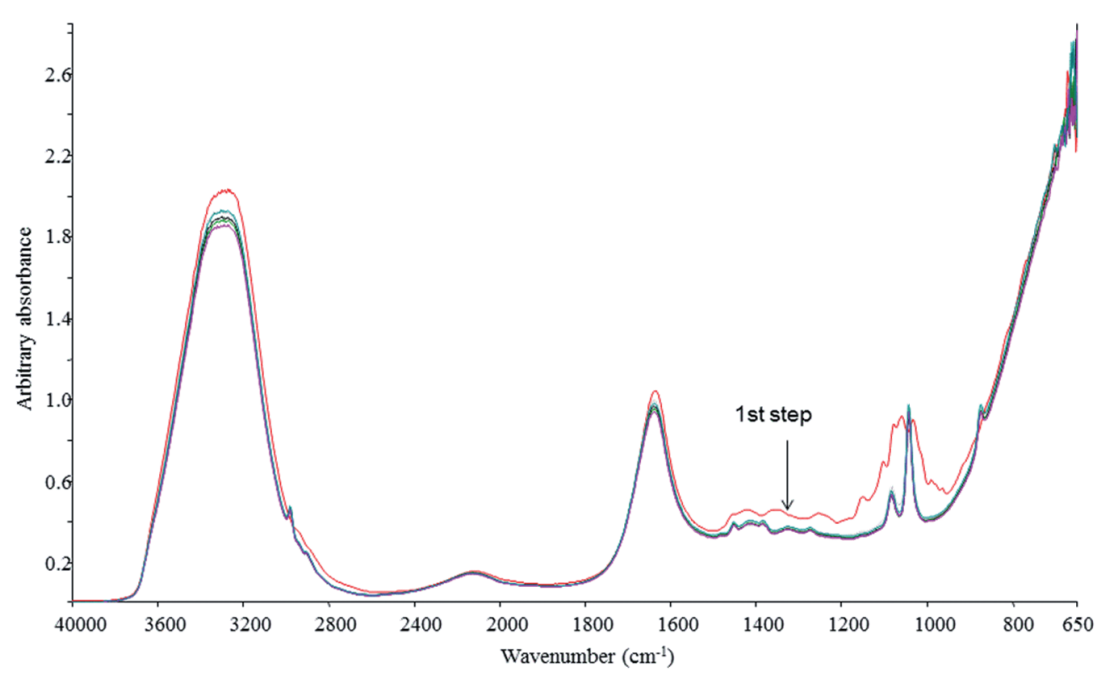

All PLS analyses for prediction of acidity, $\mathrm{pH}$, organic acids, ${ }^{\circ}$ Brix, sugars, alcohols, TPC and anthocyanin content were performed by relating FTIR spectral data of 35 wine samples as $x$ variables and the data of other parameters obtained with analytical methods as $y$ variables. Spectral filtering techniques such as WCS, WOSC and OSCW, firstand second-order derivation were all employed to mid-IR spectra before the PLS regression analysis. The best results were obtained from the second-order derivation for the quantification of chemical parameters except for alcohols. However, WOSC technique provided the best results for alcohols including ethanol and glycerol. Table 3 shows the results of statistical analysis for developed PLS models. Only selected PLS plots are shown from each group as there are 18 predicted parameters (Fig. 4). RMSE values of the models are generally low indicating the success of the models. Only the RMSE of the model for succinic acid is slightly high but could be regarded as tolerable.

The model for acids ( $\mathrm{pH}$, total acids and individual organic acids) contains 15 significant components (PCs) explaining $98.1 \%$ of the total variation of acidity $(y)$. Crossvalidation $R^{2}\left(R_{\mathrm{cv}}^{2}\right)$ of 0.58 is not very high but acceptable. a

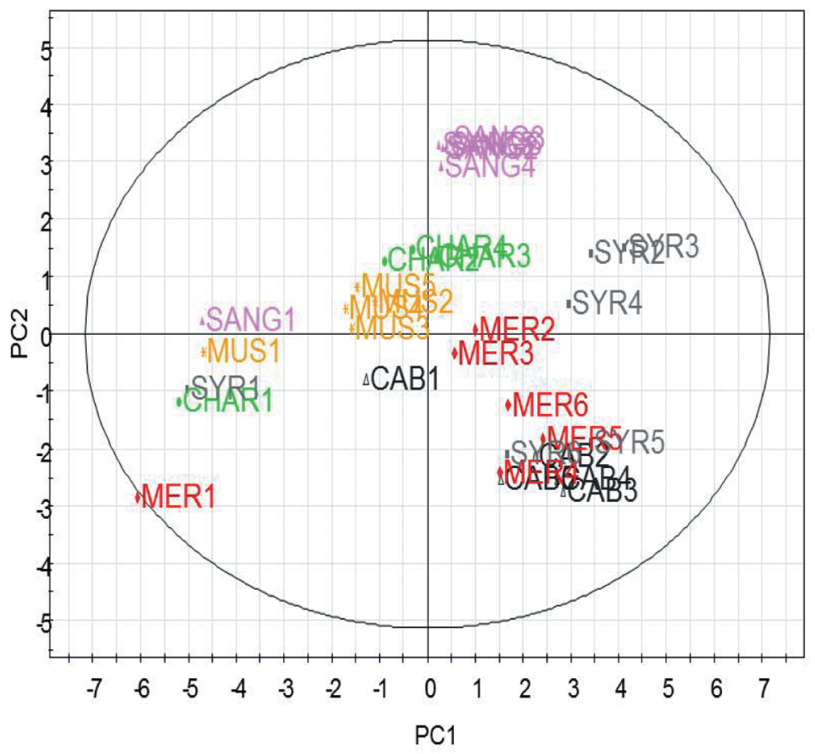

b

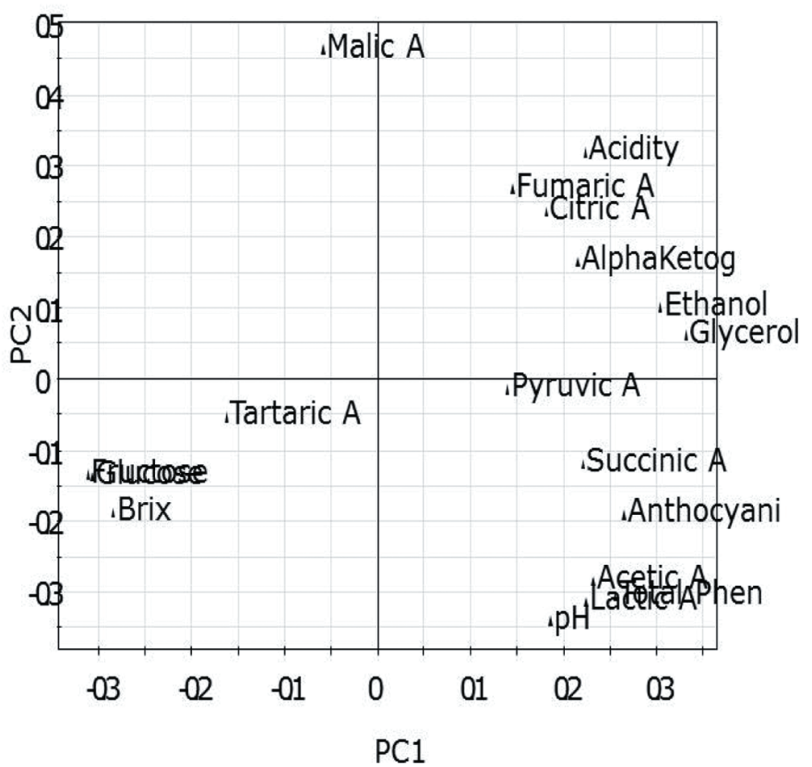

FIG. 3. PRINCIPAL COMPONENT ANALYSIS SCORE PLOT (A) AND LOADING PLOT (B) BASED ON CHEMICAL-CHROMATOGRAPHIC DATA OF WINE SAMPLES GROUPED ACCORDING TO PROCESS STEPS 
TABLE 3. SUMMARY OF STATISTICAL RESULTS FOR PLS ANALYSIS OF WINE SAMPLES

\begin{tabular}{|c|c|c|c|c|c|c|}
\hline Groups of parameters & Parameters & Number of PCs & $R^{2}$ (cal) & $R_{\mathrm{cv}}^{2}$ & RMSE & Spectral filter \\
\hline \multirow[t]{11}{*}{ Acids } & Titratable acidity & 15 & 0.98 & 0.71 & 0.096 & Second derivative \\
\hline & $\mathrm{pH}$ & 15 & 0.99 & 0.62 & 0.031 & Second derivative \\
\hline & Acetic acid & 15 & 0.99 & 0.66 & 50.949 & Second derivative \\
\hline & Citric acid & 15 & 0.96 & 0.19 & 37.800 & Second derivative \\
\hline & $\alpha$-Ketoglutaric acid & 15 & 0.99 & 0.60 & 7.056 & Second derivative \\
\hline & Fumaric acid & 15 & 0.97 & 0.49 & 4.097 & Second derivative \\
\hline & Lactic acid & 15 & 0.97 & 0.54 & 87.099 & Second derivative \\
\hline & Malic acid & 15 & 0.98 & 0.67 & 151.063 & Second derivative \\
\hline & Pyruvic acid & 15 & 0.99 & 0.49 & 2.851 & Second derivative \\
\hline & Succinic acid & 15 & 0.97 & 0.58 & 419.766 & Second derivative \\
\hline & Tartaric acid & 15 & 0.99 & 0.61 & 40.979 & Second derivative \\
\hline \multirow[t]{3}{*}{ Sugars } & ${ }^{\circ}$ Brix & 4 & 0.99 & 0.92 & 0.633 & Second derivative \\
\hline & Glucose & 4 & 0.99 & 0.95 & $3,917.622$ & Second derivative \\
\hline & Fructose & 4 & 0.99 & 0.94 & $4,208.178$ & Second derivative \\
\hline \multirow[t]{2}{*}{ Alcohols } & Glycerol & 2 & 0.97 & 0.96 & 636.441 & WOSC \\
\hline & Ethanol & 2 & 0.99 & 0.99 & 0.339 & WOSC \\
\hline Phenols & Total phenolic content & 4 & 0.99 & 0.91 & 269.217 & Second derivative \\
\hline Anthocyanins & Anthocyanins in red and rose wine & 4 & 0.99 & 0.86 & 1.794 & Second derivative \\
\hline
\end{tabular}

PC, principal component; PLS, partial least square; RMSE, root mean square of error; WOSC, wavelet in combination with orthogonal signal correction.
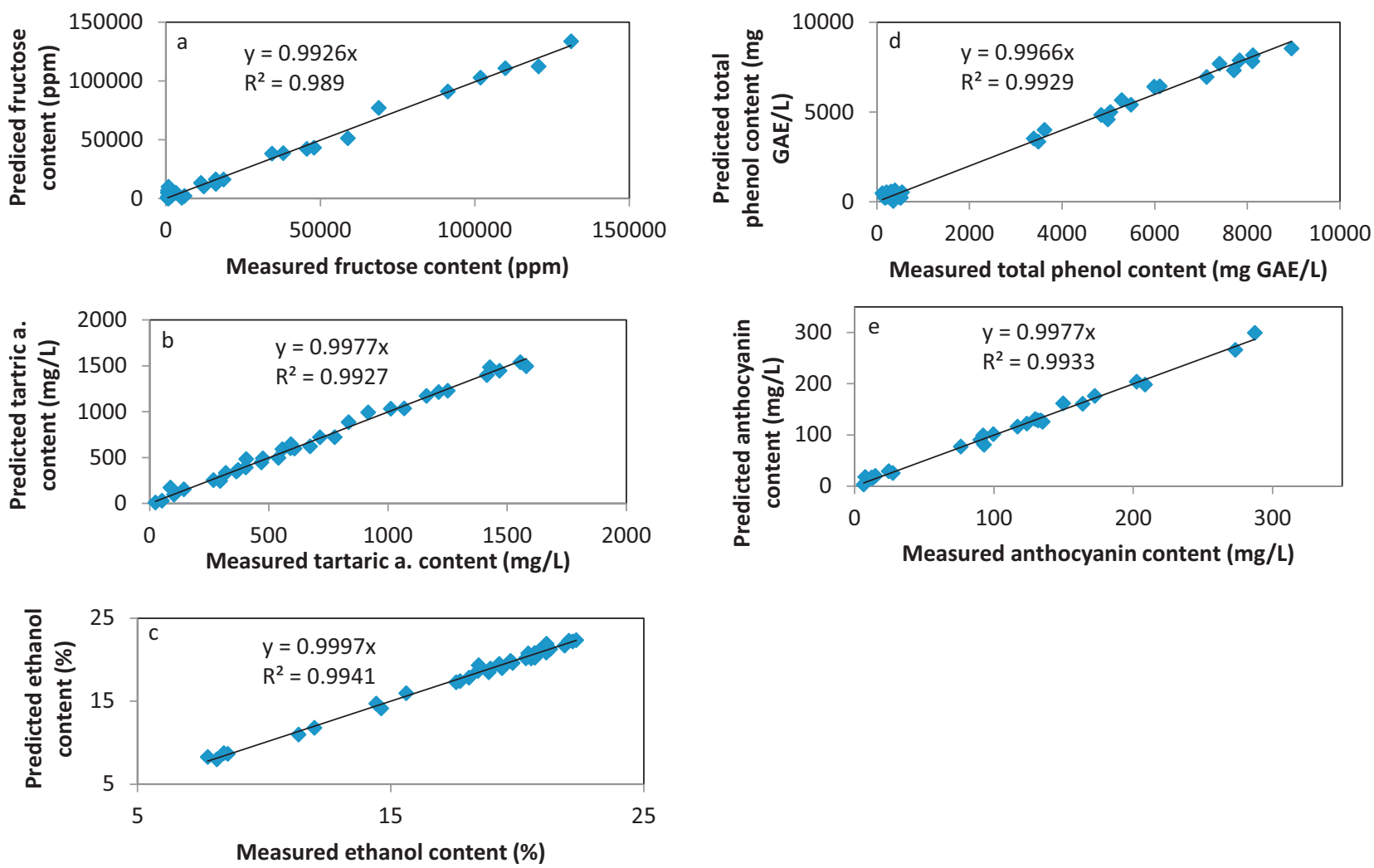

FIG. 4. PARTIAL LEAST SQUARE REGRESSION OF MEASURED VERSUS PREDICTED (A) FRUCTOSE, (B) TARTARIC ACID, (C) ETHANOL, (D) TOTAL PHENOL AND (E) ANTHOCYANIN CONTENTS OF WINE SAMPLES 
Calibration $R^{2}\left(R_{\text {cal }}^{2}\right)$ values are all greater than 0.98 . The regression coefficient of the model for selected organic acid (tartaric acid) determined with calibration set was found as 0.99 (Fig. 4a).

The model for sugars ( ${ }^{\circ}$ Brix, glucose and fructose) includes four PCs explaining $98.7 \%$ of the total variation of sugars $(y)$ with $R^{2}$ cross-validation value of 0.94 . Calibration curves for sugars have $R^{2}$ values greater than 0.98 . The PLS regression analysis for prediction of selected sugar (fructose) content from FTIR spectral data resulted in high correlation of $R^{2}$ of 0.99 (Fig. 4b), which shows an excellent prediction.

The model created for alcohol (glycerol and ethanol) prediction is composed of two PCs explaining $98.2 \%$ of total variation $(y)$ and $R^{2}$ cross-validation is 0.98 . The predictive ability of the model constructed for ethanol content with FTIR spectral data was as good as the previous models $\left(R^{2}=0.99\right)$ using a different spectral filter as shown in Fig. 4c.

TPC was also analyzed with PLS regression analysis and the created model for phenol content prediction contains four PCs explaining $99.3 \%$ of total variation $(y)$, which shows an excellent prediction with $R^{2}$ of 0.99 (Fig. 4d). This model had $R^{2}$ cross-validation of 0.91 . This figure also shows the increase of phenol content during the first three steps of the process, which is more stable in the middle and end of the process. Prediction of anthocyanin content from FTIR spectral data with the help of PLS analysis was carried out using only red and rose wines in the model. The model includes four PCs explaining 99.4\% of total variation $(y)$, which shows an excellent prediction with $R^{2}$ of 0.99 (Fig. 4e). $R^{2}$ cross-validation is 0.86 .

\section{Microbiological Monitoring of Wine Process}

The quality of wines is a direct consequence of the evolution of the microbial flora of the must during fermentation. Therefore, it is important to investigate the microbial population that play a role on fermentation of different wine samples during the whole wine process. Because $S$. cerevisiae was used for inoculation for all wine samples in the winery, all samples in each process step were investigated for yeast population. The microbial counts for each process and microbial growth are shown in Table 2.

It is observed that the yeast count is variable during the process for all wine samples. The microbial population at the beginning of the process comes from the grape and passes to the must through pressing step. Total population was suppressed by $S$. cerevisiae after inoculation for fermentation process. Our results showed that yeast count decreased during fermentation process of Cabernet and Boğazkere, whereas a decrease and then a rise of the count were observed for the other wines.
Prediction of the yeast population from IR spectroscopy did not provide accurate results as PLS model has very low $R^{2}$ values for calibration and cross-validation.

\section{CONCLUSIONS}

Several studies in the literature focused on either only the fermentation step of wine processing of only one type of wine (red, rose or white) or the final product for the prediction of several parameters by near or mid-IR spectroscopy and mostly provided accurate results (Cozzolino et al. 2006, 2011; Cozzolino and Curtin 2012; Ozturk et al. 2012). However, whole wine process from must to bottling was monitored in this study and, in general, mid-IR spectroscopic monitoring of whole wine process for red, rose and white wines provided good results with high $R^{2}(\mathrm{cal})$ and $R^{2}$ (val) values in terms of chemical parameters especially for sugars ( ${ }^{\circ}$ Brix $R^{2}[\mathrm{cal}]=0.99$, glucose $R^{2}[\mathrm{cal}]=0.99$ and fructose $R^{2}$ [cal] $\left.=0.99\right)$, ethanol $\left(R^{2}[\mathrm{cal}]=0.99\right)$ and glycerol $\left(R^{2}[\mathrm{cal}]=0.97\right)$, total phenolics $\left(R^{2}[\mathrm{cal}]=0.99\right)$ and anthocyanin $\left(R^{2}[\mathrm{cal}]=0.99\right)$ contents and titratable acidity $\left(R^{2}\right.$ [cal $\left.]=0.99\right)$ whereas $R^{2}$ (val) results were not as good as $R^{2}$ (cal) for $\mathrm{pH}$ and organic acids. However, PLS analysis did not produce accurate prediction of yeast counts when the wine samples were directly sampled. This study shows the applicability of mid-IR spectroscopy in combination with multivariate statistical analysis for a commercial wine process monitoring in order to determine the multiple components simultaneously. Therefore, mid-IR spectroscopy could be an alternative as a rapid analysis tool in monitoring the whole wine process.

\section{ACKNOWLEDGMENTS}

We would like to thank Urla Winery (Urla, Izmir, Turkey) for providing the wine samples and specialist İlknur Sen for her help in HPLC analysis. This research was funded by the Scientific Research Grants of Izmir Institute of Technology (2012-IYTE-08).

\section{CONFLICT OF INTEREST}

The authors have declared no conflict of interest.

\section{REFERENCES}

AOAC. 2000. Official methods 960.19, 962.12. In Official Methods of Analysis of AOAC International, 17th Ed., Vol. II pp. 70-75, AOAC, Gaithersburg, MD.

BAUER, R., NIEUWOUDT, H., BAUER, F.F., KOSSMANN, J., KOCH, K.R. and ESBENSEN, K.H. 2008. FTIR spectroscopy for grape and wine analysis. Anal. Chem. 80, 1371-1379. 
CASTELLARI, M., VERSARI, A., SPINABELLI, U., GALASSI, S. and AMATI, A. 2000. An improved HPLC method for the analysis of organic acids, carbohydrates, and alcohols in grape musts and wines. J. Liq. Chrom. Rel. Tech. 23, 2047-2056.

COZZOLINO, D. and CURTIN, C. 2012. The use of attenuated total reflectance as a tool to monitor the time course of fermentation in wild ferments. Food Control 26, 241-246. COZZOLINO, D., KWIATKOWSKI, M.J., PARKER, M., CYNKAR, W.U., SAMBERGS, R.G., GISHEN, M. and HERDERICH, M.J. 2004. Prediction of phenolic compounds in red wine fermentations by visible and near infrared spectroscopy. Anal. Chim. Acta 513, 73-80.

COZZOLINO, D., PARKER, M., DAMBERGS, R.G., HERDERICH, M. and GISHEN, M. 2006. Chemometrics and visible-near infrared spectroscopic monitoring of red wine fermentation in a pilot scale. Biotechnol. Bioeng. 95, 1101-1107.

COZZOLINO, D., SHAH, N., CYNKAR, W. and SMITH, P. 2011. Feasibility study on the use of attenuated total reflectance mid-infrared for analysis of compositional parameters in wine. Food Res. Int. 44, 181-186.

DI EGIDIO, V., SINELLI, N., GIOVANELLI, G., MOLES, A. and CASIRAGHI, E. 2010. NIR and MIR spectroscopy as rapid methods to monitor red wine fermentation. Eur. Food Res.

Technol. 230, 947-955.

EDELMAN, A., DIEWOK, J., SCHUSTER, K.C. and LENDL, B. 2001. Rapid method for the discrimination of red wine cultivars based on mid-infrared spectroscopy of phenolic wine extracts. J. Agric. Food Chem. 49, 1139-1145.

ESTI, M., VOLPE, G., COMPAGNONE, D., MARIOTTI, G., MOSCONE, D. and PALLESCHI, G. 2003. Monitoring alcoholic fermentation of red wine by electrochemical biosensors. Am. J. Enol. Vitic. 54, 39-45.
FERNÁNDEZ, K. and AGOSIN, E. 2007. Quantitative analysis of red wine tannins using Fourier-transform mid-infrared spectrometry. J. Agric. Food Chem. 55, 7294-7300.

JUNGMIN, L., DURST, R.W. and WROLSTAD, R.E. 2005. Determination of total monomeric anthocyanin pigment content of fruit juices, beverages, natural colorants and wines by the $\mathrm{pH}$ differential method: Collaborative study. J. AOAC Int. $88,1269-1278$.

LACHENMEIER, D.W. 2007. Rapid quality control of spirit drinks and beer using multivariate data analysis of Fourier transform infrared spectra. Food Chem. 101, 825-832.

OIV. 2009. Compendium of international methods of analysis of wines and musts. International Organization of Vine and Wine.

OZTURK, B., YUCESOY, D. and OZEN, B. 2012. Application of mid-infrared spectroscopy for the measurement of several quality parameters of alcoholic beverages, wine and raki. Food Anal. Methods 5, 1435-1442.

PATZ, C.D., BLIEKE, A., RISTOW, R. and DIETRICH, H. 2004. Application of FT-MIR spectrometry in wine analysis. Anal. Chim. Acta 513, 81-89.

SERBAN, S., DANET, A.F. and MURR, N.E. 2004. Rapid and sensitive automated method for glucose monitoring in wine processing. J. Agric. Food Chem. 52, 5588-5592.

SLINKARD, K. and SINGLETON, V.L. 1977. Total phenol analysis: Automation and comparison with manual methods. Am. J. Enol. Vitic. 28, 49-55.

TARANTILIS, P.A., TROIANOU, V.E., PAPPAS, C.S., KOTSERIDIS, Y.S. and POLISSIOU, M.G. 2008.

Differentiation of Greek red wines on the basis of grape variety using attenuated total reflectance Fourier transform infrared spectroscopy. Food Chem. 111, 192-196. 